$\operatorname{IPPP} / 02 / 44$

$\mathrm{DCPT} / 02 / 88$

24 September 2002

\title{
Diffractive Higgs Production: Myths and Reality
}

\author{
V.A. KhOZE ${ }^{a, b}$, A.D. MARTiN ${ }^{a}$ AND M.G. RYSKin ${ }^{a, b}$ \\ ${ }^{a}$ Institute for Particle Physics Phenomenology, University of Durham, DH1 3LE, UK \\ ${ }^{b}$ Petersburg Nuclear Physics Institute, Gatchina, St. Petersburg, 188300, Russia
}

\begin{abstract}
A critical comparison is made between recent predictions of the cross sections for diffractive Higgs production at the Tevatron and the LHC. We show that the huge spread of the predictions arises either because different diffractive processes are studied or because important effects are overlooked. Exclusive production offers a reliable, viable Higgs signal at the LHC provided that proton taggers are installed.
\end{abstract}

\section{Introduction}

Diffractive Higgs production may play an important role in identifying and studying a $C$ - and $P$-even, light Higgs boson at the LHC, see, for example, Ref. [1]. There exist a wide range of predictions from a variety of models for the cross section for diffractive Higgs production, which have yielded answers ranging over many orders of magnitude. One unfortunate consequence is to discredit diffractive Higgs production as a possible way to identify a Higgs boson. Here we emphasize that the huge spread of predictions is either because different diffractive processes have been considered or because important effects have been neglected. One of the aims of this note is to guide the reader through the plethora of predictions, making critical comparisons between the different approaches wherever possible.

Let us consider a light Higgs boson (with mass less than $130 \mathrm{GeV}$ ) with the dominant $H \rightarrow b \bar{b}$ decay. From an observational point of view, it is convenient to discuss three different diffractive production mechanisms, where we will use a + sign to indicate the presence of a rapidity gap. 
(a) Exclusive production: $p p \rightarrow p+H+p$

If the outgoing protons are tagged, this process has the advantage that the Higgs mass may be measured in two independent ways; first, by the missing mass to the outgoing protons and, second, by the $H \rightarrow b \bar{b}$ decay. So the signal must satisfy $M_{\text {miss }}=M_{b \bar{b}}$, with allowance for experimental resolution ${ }^{1}$. Moreover, the $b \bar{b}$ background is suppressed by a spin $\left(J_{z}=0\right)$ selection rule, which leads to a favourable signal-to-background ratio.

(b) Inclusive production: $p p \rightarrow X+H+Y$

The advantage is a much larger cross section. However, there is no spin selection rule to suppress the $b \bar{b}$ background, and the signal-to-background ratio is unfavourable. Moreover, the accuracy of the Higgs mass determination is worse, as $M_{\text {miss }}$ is not applicable.

(c) Central inelastic production: $p p \rightarrow p+(H X)+p$

There is additional radiation accompanying the Higgs in the central region, which is separated from the outgoing protons by rapidity gaps. Although this mechanism is often used for predictions, it has, in our view, no special advantages for Higgs detection.

We may regard each large rapidity gap as being generated by an effective Pomeron exchange. It may be either a QCD Pomeron, which at lowest order is a gluon-gluon state, or a phenomenological Pomeron with parameters fixed by data. The above information is summarised in Fig. 1, together with a leading order QCD diagram of each process.

(a) exclusive

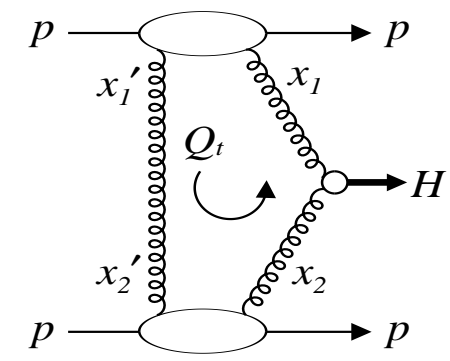

$$
M_{\text {miss }}=M_{H}
$$

$J_{z}=0$ rule for background

$$
S / B \sim 3
$$

pile-up may be overcome (b) inclusive

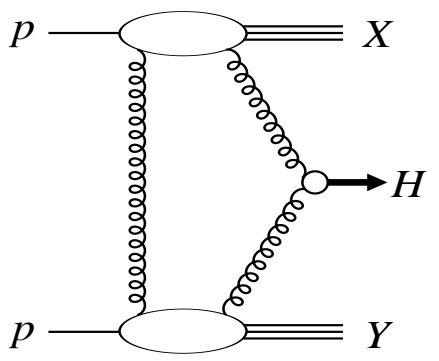

$$
\text { no } M_{\text {miss }}
$$$$
\text { no rule }
$$$$
S / B \sim 0.01
$$

pile-up problems (c) central-inelastic

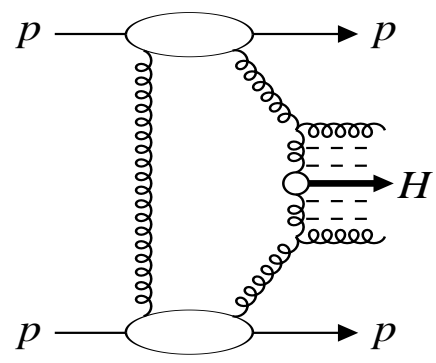

$$
\begin{gathered}
M_{\text {miss }}>M_{H} \\
\text { no rule } \\
S / B \sim 0.001
\end{gathered}
$$

pile-up may be overcome

Figure 1: Different processes for double-diffractive Higgs production in $p p$ collisions in terms of perturbative QCD. The signal-to-background ratios, $S / B$, are obtained using the mass resolutions, $\Delta M_{\text {miss }}=1 \mathrm{GeV}$ and $\Delta M_{b \bar{b}}=10 \mathrm{GeV}$ expected for experiments at the LHC [1]. Pile-up refers to the multiple interactions per bunch crossing at the LHC.

\footnotetext{
${ }^{1}$ This way to identify a light Higgs boson in Run II of the Tevatron was proposed in Ref. [2]. The experimental issues concerning the LHC measurements are covered in [1].
} 
Recall that, at medium and high luminosity at the LHC, the recorded events will be plagued by overlap interactions in the same bunch crossing. For example, at the medium luminosity of $10^{33} \mathrm{~cm}^{-2} \mathrm{~s}^{-1}$, an average of 2.3 inelastic events are expected for each bunch crossing. Hence the rapidity gaps occurring in one interaction may be populated by particles created in an accompanying interaction. It is, however, possible to use detector information to locate the vertices of the individual interactions and, in principle, to identify hard scattering events with rapidity gaps. For the exclusive and central inelastic processes of Figs. 1(a) and 1(c), the use of proton taggers makes it much more reliable to select the rapidity gap events ${ }^{2}$.

We note that the significance of a signal is only increased if the background-to-signal ratio is decreased by more than the signal. This does not happen for either (b) inclusive production or (c) central-inelastic production. Indeed, the corresponding effective (Pomeron-Pomeron) luminosities are orders of magnitude smaller than the gluon-gluon luminosity which governs the conventional totally inelastic Higgs production signal, while the background-to-signal ratio is decreased by at most a factor of two due to the lower hadronic multiplicity.

In principle, factorization does not hold for the diffractive processes; it can only occur by chance. It is spoilt by the Pomeron and Reggeon cut contributions, and by QCD radiation. Moreover, the comparison [3] of CDF dijet and HERA diffractive data demonstrates a strong violation of factorization [4]. Nevertheless, the existing approaches may be classified as factorizable or non-factorizable. Some authors $[5,6,7,8]$ use factorization à la Ingelman-Schlein model [9] and then introduce a normalising factor to account for the non-factorizable nature of the process.

\section{Survival probability of the rapidity gaps}

The cross sections for processes with rapidity gaps are reduced by the probabilities of the gaps not to be populated by, first, the gluon radiation associated with a QCD Pomeron and/or the hard $g g \rightarrow H$ subprocess and, second, by secondaries produced in the soft rescattering of the spectator partons. We denote these survival probabilities by $T^{2}$ and $S^{2}$ respectively. The probability amplitude $T$, not to radiate, can be calculated using perturbative QCD. The expression for $T$ has the familiar double-logarithmic Sudakov form. In fact it is possible to include the next-to-leading (single log) corrections in $T$ [11]. Note that the amplitude $T$ plays a crucial role in providing the infrared convergence of the loop integral over the $t$ channel gluon transverse momentum $Q_{t}$. On the other hand the survival factor, $S^{2}$, to soft rescattering cannot be calculated perturbatively. The presence, and the value, of $S^{2}$ has been checked experimentally by comparing the diffractive cross section in deep inelastic reactions at HERA (where $S$ is close to 1 , due to the absence of soft spectators in the virtual photon) with the cross

\footnotetext{
${ }^{2}$ In addition to helping to select events with rapidity gaps, it may be possible to use the proton taggers to measure the approximate position of the vertex of the event, although the accuracy is expected to be $\pm 3-5 \mathrm{~cm}$ at best.
} 
section of diffractive dijet production at the Tevatron, for which it turns out that $S^{2} \sim 0.1$ [3]. Theoretical predictions of the survival factor, $S^{2}$, can be found in Refs. [12, 13, 14]. Note that the factor $S^{2}$ is not a universal number. It takes account of soft rescattering in both the initial and final state interactions. Therefore the value of $S^{2}$ depends on the initial energy and the particular final state. Clearly, the presence of such a factor violates factorization [15].

It is informative to digress for a moment and to note that the large rapidity gaps may also be produced by electroweak interactions, as well as by QCD. Exclusive Higgs production can proceed by $\gamma \gamma$ fusion $^{3}$, while the inclusive reaction can proceed via weak boson fusion. As the photon and the $W$ boson are point-like colour singlets, there is no $T^{2}$ factor in these reactions. However, we still have to allow for the survival factor, $S^{2}$, to soft rescattering. The process $\gamma \gamma \rightarrow H$ is dominated by photons with very small transverse momenta, which corresponds to the interaction occurring mainly at large impact parameters. There are two consequences. First, the factor $S^{2} \simeq 1$ (or, to be precise, 0.86 at the LHC, see [16]) and, second, there is almost no interference between the QCD and $\gamma$ exchange amplitudes. In the case of weak boson fusion the interference is also suppressed, but now due to the much larger transverse momentum (about $M_{W} / 2$ ) transferred to the Higgs boson. The survival factor $S^{2}$ is comparable to that for the QCD-induced reaction; in fact a bit larger, since the $W$ bosons are emitted from valence quarks which are more concentrated in the component of the proton wave function which has smaller absorption $[11,13,16]$.

\section{Exclusive diffractive Higgs production}

The first QCD-based calculation of double-diffractive Higgs production was performed by Bialas and Landshoff [17]. In terms of QCD the Higgs boson is produced by gluon-gluon fusion. The colour of these $t$ channel gluons is screened, at leading order, by an accompanying $t$ channel gluon exchange between the incoming protons, as shown in the diagrams in Fig. 1. The screening is necessary as colour cannot be transferred across a rapidity gap; colour flow would populate the gap with secondaries via hadronization. Thus we need two-gluon colour-singlet exchange across the gaps. Effectively in this approach, we may regard the Higgs as being produced by the fusion of two 'Low-Nussinov'-like [18] di-gluon Pomerons. A more precise understanding of this statement will emerge as we discuss the different production mechanisms below.

Bialas and Landshoff [17] did not include in the calculation the probabilities $T^{2}$ and $S^{2}$ that the rapidity gaps survive QCD radiation and soft rescattering respectively, although they left open the possibility that extra radiation may result in an inclusive process. However, they did not quantify this effect. A number of authors have adopted a similar approach to calculate the cross section for Higgs production with large rapidity gaps. Some of the most recent calculations are listed in Table 1.

\footnotetext{
${ }^{3}$ The known $\gamma \gamma$ fusion process provides a lower limit for exclusive Higgs production; see, for example, [16] and references therein.
} 
We start the discussion of the results shown in Table 1 with the calculation of the exclusive double-diffractive cross section by Levin [20]. He assumed the survival probability to soft rescattering $S^{2}=0.1$. To account for QCD radiation he multiplied the final result by an effective $T^{2}$ factor which was estimated phenomenologically assuming a Poisson probability $\exp (-\bar{n})$, where $\bar{n}$ is the mean multiplicity of mini-jets produced in hadron interactions with energy $\sqrt{s} \simeq M_{H}$. This assumption overestimates the survival factor $T^{2}$ in comparison with the perturbative QCD calculation, since instead of getting the double-logarithmic Sudakov-like suppression, his probability $\exp (-\bar{n})$ corresponds to a single logarithm.

In the calculation by Cudell and Hernandez [21], both the soft $S^{2}$ and hard $T^{2}$ survival factors were neglected. In addition to the pure exclusive process, inclusive events where an incoming proton dissociates into $N^{*}$ resonances were allowed, so the predicted cross section becomes larger. A crucial point, both in this calculation and in that of Levin, is the normalization of the two-gluon exchange amplitude. Without the double-logarithmic $T$ factor inside the loop integration over the gluon transverse momentum $Q_{t}$, the integral is infrared divergent. To obtain a finite result the authors have to choose an infrared cut-off or to introduce a finite mass for the gluon. The value of the cut-off, or mass, is tuned to reproduce the total $p p$ cross section, $\sigma_{\text {tot }}$, in terms of the Low-Nussinov two-gluon Pomeron exchange. It has been noted [23, 22] that the use of such a prescription further overestimates the Higgs production cross section. Indeed, in terms of $Q_{t}$ factorization, the Higgs production forward amplitude is of the form

$$
\mathcal{M}_{\mathrm{Higgs}}=A \pi^{4} \int \frac{d Q_{t}^{2}}{Q_{t}^{4}} f_{g}\left(x_{1}, x_{1}^{\prime}, Q_{t}^{2}\right) f_{g}\left(x_{2}, x_{2}^{\prime}, Q_{t}^{2}\right)
$$

where the factor $A$ represents the $g g \rightarrow H$ vertex and $f_{g}\left(x, x^{\prime}, Q_{t}^{2}\right)$ is the unintegrated skewed gluon density. The unintegrated gluon density embodies the $T$ factor $[10,11]$ which accounts for the fact that the gluon which participates in the hard $g g \rightarrow H$ subprocess remains untouched in the evolution from $Q_{t}$ up to the hard scale, $\sim M_{H} / 2$; this hard scale is an implicit variable in the $f_{g}$ in (1). Similarly, via the optical theorem, we may express the total cross section in terms of two-gluon exchange

$$
\sigma_{\text {tot }}=\frac{\pi^{3}}{2} \int \frac{d Q_{t}^{2}}{Q_{t}^{4}} f_{g}\left(x, x, Q_{t}^{2}\right) f_{g}\left(x, x, Q_{t}^{2}\right)
$$

where $x \lesssim 2 Q_{t} / \sqrt{s}$, as follows from the internal kinematics of the process, and where the implicit scale in $f_{g}$ is now $\sim Q_{t}$. At first sight it appears that (2) will give a precise normalisation of the Higgs cross section, via (1). However, in addition to the different implicit scales, the typical values of $x$ sampled in (2) are about two orders of magnitude smaller than the values of $x_{i} \sim M_{H} / \sqrt{s}$ sampled in (1). Since $f_{g}$ grows as $x$ decreases and since $\sigma_{\text {Higgs }} \propto\left|f_{g}\right|^{4}$, this normalisation considerably overestimates the cross section for Higgs production. Despite the fact that $S^{2}$ and $T^{2}$ factors were included in the prediction of the cross section given in [20], the result is close to that of [21]. One reason is that these small survival factors are compensated by the use of a larger value ${ }^{4}$ of $\alpha_{S}$ in the $g g \rightarrow H$ vertex.

\footnotetext{
${ }^{4}$ It is argued in Ref. [20] that, instead of the conventional $\alpha_{S}\left(M_{H}\right)$, a much larger QCD coupling (at low
} 
The reliability of the prediction of the diffractive production of the Higgs boson can be checked experimentally by measuring the much larger cross section for double-diffractive central production of a pair of high $E_{T}$ jets $[11,22]$. The amplitude for this process has the same structure as (1), with the $g g \rightarrow H$ vertex replaced by the matrix element of the $g g \rightarrow g g$ subprocess. The original calculation of dijet production was performed by Berera and Collins [24]. The result [23], with $S^{2}$ and $T^{2}$ factors neglected and normalised to $\sigma_{\text {tot }}$, is about $5600 \mathrm{nb}$ for CDF dijets at the Tevatron energy, in contrast with the experimental upper limit of less than $3.7 \mathrm{nb}$ at $95 \%$ confidence level [25]. The huge difference originates from the product of three factors - the survival factors $S^{2} \simeq 0.05-0.1$ and $T^{2} \simeq 0.1-0.2$ should be included in the prediction, and the normalisation should be reduced by about a factor of 10 , since (2) should be compared to (1) at much lower $x$. Indeed Berera and Collins [24] had noted that the survival factors should be computed before their leading order calculation is compared with data. In fact, when account is taken of the survival factors, our perturbative approach [11, 22] leads to the prediction of about $1 \mathrm{nb}[26]$ for the exclusive production of dijets corresponding to the kinematics $^{5}$ of the CDF dijet search [25], which leads to a dijet bound of less than $3.7 \mathrm{nb}$.

Let us return to the discussion of the predictions listed in Table 1. Since Cudell and Hernandez [21] do not include the $S^{2}$ and $T^{2}$ survival factors, and apply a $\sigma_{\text {tot }}$ normalisation, we may expect that the dijet cross section would be overestimated by a factor of about 1000 . Levin [20] includes estimates of the $S^{2}$ and $T^{2}$ factors and, following his prescription, we would expect a dijet cross section of about $1000 \mathrm{nb}$, which is still much larger than the experimental limit. There is no simple way of using these dijet overshoot factors to correct the predictions for Higgs production given in Refs. [20, 21]. We cannot simply scale down the predictions by dividing by the overshoot factors. The correction factor has, first, an energy dependence arising from the effective gluon density normalised to $\sigma_{\text {tot }}$ and, second, due to the energy dependence of the soft survival factor $S^{2}$. Moreover, the QCD radiative effects described by the $T$ factor depend strongly on the hard scale, and are quite different for dijet production, with jets of $E_{T} \sim 7-10 \mathrm{GeV}$, and Higgs production with scale $M_{H} / 2 \sim 60 \mathrm{GeV}$.

Instead of fixing the normalisation of the prediction for exclusive Higgs production by using $\sigma_{\text {tot }}$, a more reliable method is to use the gluon density given by global parton analyses and to include the Sudakov-like survival factor $T=\exp \left(-\mathcal{S}\left(Q_{t}^{2}, M_{H}^{2}\right)\right)$ inside the loop integral over $Q_{t}$ in (1) [27]. This factor provides the infrared stability of the integral, while the known gluon distribution fixes the normalisation. More recently, the method has been further improved [11, 16]. First, the skewed effect is included (using the prescription of Refs. [28, 29]), that is the effect due to unequal longitudinal momentum fractions carried by the left and right $t$ channel gluons in Fig. 1(a): explicitly, we have $\left(x_{i}^{\prime} \simeq Q_{t} / \sqrt{s}\right) \ll\left(x_{i} \simeq M_{H} / \sqrt{s}\right)$. Second, the NLO corrections to the $g g \rightarrow H$ vertex, and the next-to-leading correction to the double-logarithmic

scale, $\sim 1 \mathrm{GeV}$ ) is to be taken. However, the high-order evolution of the Higgs vertex confirms the former choice [11].

${ }^{5}$ The accuracy of the theoretical prediction for $E_{T}>7 \mathrm{GeV}$ jets at the Tevatron energy is far from as good as the factor of 2 uncertainty claimed for dijets of mass $M(j j) \sim M_{H}$ at the LHC. The contribution from the low $Q_{t}$ domain is less under control for the CDF kinematics of Ref. [25]. 
$T$ factor (that is the single log term in $T$ ), are included ${ }^{6}$. This is the method used for obtaining the numbers quoted for the third entry [16] in Table 1.

The most delicate point, in the prediction of the cross section for diffractive Higgs production, is the calculation of the probability, $S^{2}$, that the rapidity gaps survive the soft rescattering. $S^{2}$ cannot be determined using perturbative QCD and non-perturbative techniques have to be applied. To improve the accuracy of the prediction of $S^{2}$, a detailed analysis ${ }^{7}$ of all available soft high energy $p p$ and $p \bar{p}$ data was performed [12]. Using the results of this analysis it is possible to compute the soft survival factor $S^{2}$ for a complete range of diffractive processes. The factors for Higgs production are given in Refs. [11, 12, 16]. For exclusive Higgs production at the LHC the soft survival factor $S^{2}$ is found to be 0.02. After all the above effects are included, the uncertainty in the prediction of the cross section, $\sigma(p p \rightarrow p+H+p) \simeq 3 \mathrm{fb}$, is estimated to be about a factor of two [1].

\section{Inclusive diffractive Higgs production}

If we allow the protons to dissociate, but still keep the rapidity gaps on either side of the produced Higgs boson, then we enlarge the cross section by a factor of $3-10$, depending on the range of masses allowed for the dissociation $[17,21,11,20,16]$. In addition to the larger available phase space for inclusive kinematics, also the gap survival factor is larger; in fact using the formalism of Ref. [12] we find $S_{\text {incl }}^{2} \simeq 0.1$ at the LHC, while for exclusive and central inelastic production we have $S^{2} \simeq 0.02$. However, we lose all the advantages of exclusive doublediffractive Higgs production. In particular, we lose the good missing mass resolution provided by the proton tagger, the equality $M_{\text {miss }}=M_{b \bar{b}}$ from the $H \rightarrow b \bar{b}$ decay, and the suppression of the $b \bar{b}$ QCD background and of the pile-up events. We therefore do not discuss this process any further here.

\section{Central inelastic Higgs production}

So far we have considered processes where there are no secondaries accompanying Higgs production in the central rapidity region. By 'central inelastic Higgs production' we mean that secondaries are allowed in some central rapidity interval. Two contributions to the process are sketched in Fig. 2. As we shall see in a moment, we may call diagrams 2(a) and 2(b) lower and higher order $\alpha_{S}$ contributions respectively. In fact, much attention is paid in the literature to Higgs production in Pomeron-Pomeron inelastic collisions, Fig. 2(b), which, in our notation, corresponds to the higher order $\alpha_{S}$ contribution to central inelastic production. So we discuss

\footnotetext{
${ }^{6}$ Note that the gluon with $x^{\prime} \simeq 0$ is almost 'at rest' and practically does not radiate. Thus, the QCD radiation is associated with the hard $g g \rightarrow H$ subprocess.

${ }^{7}$ The data were analysed in terms of a two-channel eikonal model, which also incorporated high mass diffraction and $\pi$-loop insertions in the Pomeron trajectory (to describe better the periphery of the proton).
} 
(a) Central-inelastic (LO)

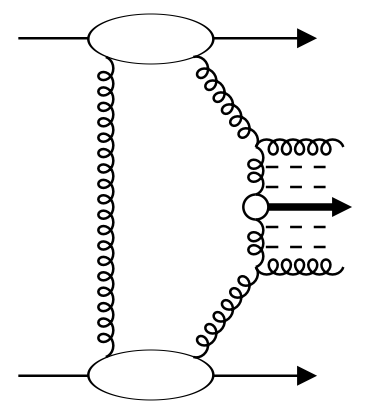

(b) soft $\mathbb{P} I P$

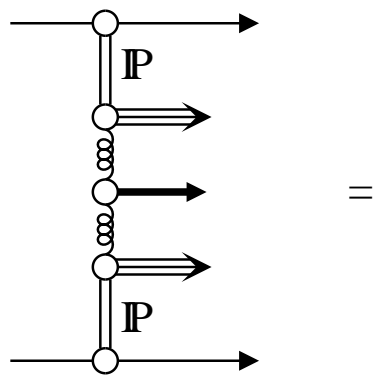

(c) Low-Nussinov

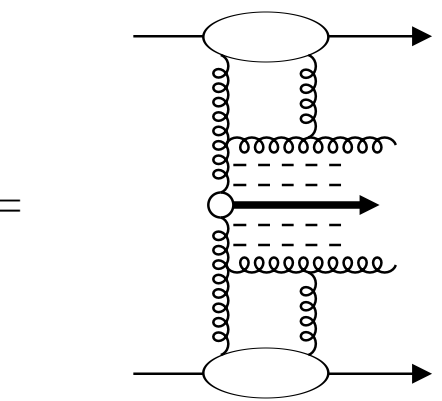

Figure 2: Central inelastic double-diffractive production, in which the Higgs boson (shown by the bold central arrow) is accompanied by gluon emission in the central rapidity region. Diagram (b) shows the contribution from Pomeron-Pomeron inelastic collisions in which the Pomeron remnants populate the central region. Diagram (c) shows the Pomeron-Pomeron production process from a QCD viewpoint, in which each Pomeron is represented by two-gluon exchange. Diagrams 2(a) and 2(b,c), respectively, may be regarded as lower and higher order $\alpha_{S}$ contributions to central inelastic production.

this first. The cross section for Higgs production by Pomeron-Pomeron collisions is larger than for exclusive diffractive production, but still much smaller than that for the normal inclusive production, $p p \rightarrow H X$. The expected signal-to-background ratio is practically the same as for normal inclusive production but at a lower energy, corresponding to the Pomeron-Pomeron energy as measured by the missing mass method. We have effectively degraded the LHC energy down to energies comparable to the Tevatron! Of course, the luminosity of the LHC is larger than that of the Tevatron. However, the effective Pomeron-Pomeron luminosity contains its own small factors. The only advantage ${ }^{8}$ of Higgs production by Pomeron-Pomeron inelastic collisions, in comparison to normal inelastic production, at the LHC, is the possibility to use proton taggers to avoid pile-up problems (associated with multiple interactions in each bunch crossing).

Usually the cross section for central inelastic production is estimated using the factorization hypothesis, à la Ingleman-Schlein [9]. To account for the probability $S^{2}$ that the rapidity gaps survive the soft rescattering (which violates factorization), the predictions are normalised to the observed rate of central inelastic double-diffractive dijet production at the Tevatron [25]. From a QCD viewpoint, the soft Pomeron-Pomeron interaction, Fig. 2(b), should be regarded as Fig. 2(c) where the soft Pomerons are replaced by (Low-Nussinov) two-gluon exchange. We note that Fig. 2(c) contains an extra factor of $\alpha_{S}$, as compared to Fig. 2(a). Of course, this coupling occurs at low scale, but nevertheless we should not be surprised when we find that the contribution of Pomeron-Pomeron collisions, Fig. 2(c), is less than that of central inelastic production, Fig. 2(a). For example, in Ref. [5] the cross section corresponding to Fig. 2(b,c) was calculated using the $\mathrm{H} 1$ parameterisation of the Pomeron flux and structure function [30].

\footnotetext{
${ }^{8}$ Also, $b$-tagging may be easier due to the lower mean multiplicity of soft secondaries, see also [6].
} 
In the absence of dijet data at the LHC energy, the LHC prediction of [5] is presented without accounting for the $S^{2}$ factor. If we multiply this result by the same $S^{2}=0.02$ as in Refs. [12, 16] we obtain $\sigma(\mathrm{C}$.inel $) \simeq 6 \mathrm{fb}$, which is an order of magnitude smaller than $50 \mathrm{fb}$ - the cross section corresponding to Fig. 2(a).

Central inelastic production, Fig.1(c), may be regarded as higher-order QCD radiative corrections to exclusive Higgs central production (Fig. 1(a)). Allowing QCD radiation, in a central rapidity interval around the Higgs boson, increases the probability of gap survival, but weakens the potential of the $T$ factor to provide infrared convergence of the loop integral over $Q_{t}$. The cross section is increased, with the extra contribution coming from the low $Q_{t}$ region. In Ref. [16], results were focused on central inelastic production allowing radiation only in a relatively small central rapidity interval, $\delta \eta$. In this case, the residual $T$ factor is still able to ensure infrared convergence of the loop integral. If proton taggers are installed, then the mass of the central system (that is the Higgs plus accompanying radiation) can be measured by the missing mass method. For the large masses, up to $M_{\text {miss }}=1.4 \mathrm{TeV}$ that were considered in Ref. [7], the $T$ factor approaches unity and almost any QCD radiation is allowed. In these circumstances there is no convergence of the $Q_{t}$ integral, and the only possibility is to normalise the prediction to $\sigma_{\text {tot }}$, recall (1) and (2). The typical values of $x$ sampled in (1) are $x \sim M_{H} / \sqrt{s} \sim 0.01$ at the LHC. To evaluate (2) in a comparable $x$ domain, we use the value of $\sigma_{\text {tot }}$ at a much lower (CERN-ISR) energy. Based on this normalisation, and including a soft survival factor $S^{2}=0.02$, we predict a central inelastic cross section of $50 \mathrm{fb}$, which is to be compared to the $320 \mathrm{fb}$ predicted in Ref. [7]. Strictly speaking, the $320 \mathrm{fb}$ in [7] was calculated for Pomeron-Pomeron inelastic collisions, Fig. 2(b). For comparison, if for this latter process we were to use the Donnachie-Landshoff [31] parameterization for the Pomeron flux, the Pomeron structure function as measured by [30], and the known soft survival factor $S^{2}$, we would obtain $1.7 \mathrm{fb}$ at the LHC. However, in [7] the cross section was normalized using the CDF dijet data [25] at the Tevatron, for which the dominant contribution comes from central inelastic diagrams of the type Fig. 2(a); so comparison with $50 \mathrm{fb}$ is more relevant. Note that the dijet mass distributions are driven (modulo detector effects) by the logarithmic structure of the available longitudinal phase space and by the value of the Pomeron intercept, and so lead to a similar mass distribution for Figs. 2(a) and 2(b).

The residual discrepancy between $320 \mathrm{fb}$ and $50 \mathrm{fb}$ may be traced, first, to the fact that the same gap survival factor, $S^{2}$, is assumed in [7] for LHC and Tevatron energies, whereas it is expected $[12,13,14]$ that $^{9}$

$$
S_{\mathrm{LHC}}^{2} / S_{\mathrm{CDF}}^{2} \simeq 0.4
$$

Secondly, a smaller slope, $\lambda_{H}=2 \lambda_{\text {dijet }} / 3$, is used for Higgs, as compared to dijet, production; see eq. (1) of [7]. Neglecting the Pomeron slope, $\alpha_{P}^{\prime}$, the cross section is proportional to $1 / \lambda^{2}$. Finally, at the Tevatron energy, an extra contribution to Fig. 2(b) comes from Reggeon-Reggeon

\footnotetext{
${ }^{9}$ The decrease of $S^{2}$ with collider energy reflects the rise of the total interaction cross section, and is in agreement with the D0 and CDF data for the production of jets separated by rapidity gaps, measured at 630 and $1800 \mathrm{GeV}$ [32].
} 
and Pomeron-Reggeon exchange interactions. Allowing for all these effects would decrease the predicted Higgs cross section of $320 \mathrm{fb}$ by about a factor in the region of $5-10$, bringing the cross section of Ref. [7] into general agreement with our central inelastic prediction at the LHC. For the Tevatron energy, instead of the number given in Ref. [6] we have entered the later prediction of Ref. [7].

For the central inelastic configuration, it was claimed in [7] that, by tagging the outgoing protons, and by measuring the jets accompanying the Higgs, it is possible to obtain a good missing mass resolution for the Higgs. Unfortunately this is only true for a centrally produced system of $M_{\text {miss }}$ close to $M_{H}$, which corresponds to a very small fraction of the events, comparable to the number for exclusive production. Moreover, for the reasons listed above, the cross section was overestimated.

The Pomeron-Pomeron approach of Cox et al. [5] is close to that of Boonekamp et al. [6]. The main difference is that, instead of using a soft Pomeron intercept $\alpha_{P}(0)=1.08$, a larger intercept $\alpha_{P}(0) \sim 1.2$ was used, as given by the $\mathrm{H} 1$ diffractive deep inelastic data. Again the prediction is normalised to the CDF dijet data [25]. Therefore the prediction at the Tevatron energy is reasonable. Cox et al. [5] use the same parameters for the Higgs and dijet production amplitudes. Moreover, they use the H1 analysis of diffractive data to specify the flux and the gluon structure of the Pomeron. They find that their normalisation is equivalent to a soft survival factor of $S^{2} \simeq 0.15$ at the Tevatron. The theoretical expectation for $S^{2}$ is about 0.05. This implies that a significant part of the cross sections must come from the larger Fig. 2(a) contributions, rather than Fig. 2(b), to compensate for the smaller value of $S^{2}$.

Unlike all the previous approaches, the predictions of the Soft Colour Interaction (SCI) model of Enberg et al. [19, 33] are obtained from Monte Carlo simulations, rather than from an analytic approach. The model assumptions on soft interaction are implemented in PYTHIA [34] and embody the possibility of soft spectator rescattering and initial state QCD radiation. The SCI model effectively incorporates the $S^{2}$ and $T^{2}$ survival factors generated within the framework of the PYTHIA Monte Carlo [34]. Rapidity gaps are produced in the model [19, 33] by additional soft colour interactions in the final state, which are contrived to screen the colour flow across the gaps. The strength of these extra soft colour interactions was tuned to reproduce the diffractive deep inelastic data obtained at HERA. It was demonstrated that the model, with the same parameters, describes reasonably well the single diffractive processes observed at the Tevatron.

However, the generator was created to simulate inelastic processes. It operates by starting from the hard subprocess and generates the parton showers by backward evolution. The generator never accounts for the important coherence between different parton showers, nor for the colourless nature of the initial particles. The incoming protons are just considered as a system of coloured partons and only the overall colour charge is conserved. As a consequence, the probability not to emit additional secondary jets (and so to reproduce an exclusive process) turns out to be negligibly small. In particular, such a generator is unable to reproduce the elastic cross section. Originally these generators create many secondary minijets at the parton 
shower stage and the probability to screen all these minijets by colour interchange is extremely low. Such generators were not constructed to reproduce exclusive processes, where the colour coherence effects and colourless nature of the incoming hadrons are important. For this reason we believe that the extremely low limit for the exclusive $p p \rightarrow p+H+p$ cross section, which would follow from such an approach, would not be trustworthy.

It is informative to note that, in our perturbative QCD approach [11], the effective Pomeron or two-gluon exchange has relatively compact transverse size. The Sudakov-like $T$ factor occurs inside the loop integral over $Q_{t}$ and, in this way, the large-size (small $Q_{t}$ ) component of the Pomeron is strongly suppressed by QCD radiative effects. When the two-gluon system forms a large-size colour-dipole it emits numerous secondary gluon jets which completely fill the rapidity gap. There is a vanishing small probability $T^{2}$ for the gap to survive such emissions. The main contribution to the loop integral comes from relatively large $Q_{t}$ in the region of the saddle point $Q_{S}$. The value of $Q_{S}$ grows with both $M_{H}$ and the collider energy $\sqrt{s}$. For a Higgs of mass $M_{H}=120 \mathrm{GeV}$ produced at the LHC, the transverse size of the exchange is $r_{P} \sim 1 / Q_{S} \sim$ $0.1 \mathrm{fm}$. On the contrary, in the approaches of references [5, 6, 7, 33, 19], a soft large-size Pomeron is exchanged across the rapidity gaps with transverse size $r_{P} \sim r_{\text {proton }} \sim 1 \mathrm{fm}$. This could cause a much stronger Sudakov suppression if it were to be calculated by perturbative QCD.

Another consequence of the small size of the perturbative Pomeron concerns the validity of the $J_{z}=0$ selection rule for the semi-forward hard diffractive production amplitudes. Recall that this rule plays a crucial role in the suppression of the QCD background ${ }^{10}[26,1]$. In the exact forward direction, $J_{z}=0$ by virtue of angular momentum conservation. However, violation of this rule can come from orbital angular momenta, $r p_{i t}$, where $p_{i t}$ is the transverse momentum of the leading proton and $r \sim 1 / Q_{t}$ is the transverse size of the Pomeron. Therefore the admixture of the $\left|J_{z}\right|=2$ state is strongly suppressed for the small-size Pomeron-exchange occurring in the exclusive amplitude [36]. On the other hand, for C-inelastic production, where the $T$ factor becomes inactive and we deal with a large-size Pomeron, we lose the $J_{z}=0$ selection rule and, as a result, have a much larger background. The same is true for Monte-Carlo-based models. The soft colour interaction, which screens the colour across the gap, takes place at large distances and therefore we have no $J_{z}=0$ selection rule. So the expected signal-to-background ratio is small.

\section{Résumé}

We compiled a representative range of different predictions of the cross sections for diffractive production of a Higgs boson of mass about $120 \mathrm{GeV}$ at the Tevatron and LHC. We critically

\footnotetext{
${ }^{10}$ Due to the QCD factorization of soft gluon emission (see, for example, Ref. [35]) the $J_{z}=0$ selection rule is still valid, and suppresses the $b \bar{b}$ background, even beyond leading order, arising from events where the $b \bar{b}$ pair is accompanied by one or more soft gluons [1]. Hence the QCD-induced $b \bar{b}$ background is expected to be suppressed both for the exclusive process and for low mass central-inelastic production where the missing mass $M_{\text {miss }} \equiv M_{P P}$ is close to $M_{H}$.
} 
compared the wide range of predictions and explained the origin of the differences. In summary, the wide spread of predictions occurs either because different processes have been considered or because important effects have been neglected.

The cross sections for inclusive and central inelastic diffractive Higgs production are larger than for exclusive production. However, for these non-exclusive processes it is hard to suppress the QCD $b \bar{b}$ background and the signal-to-background ratio is small. Second, we cannot improve significantly the accuracy of the measurement of the mass of the Higgs boson by tagging the forward protons and measuring the missing mass.

On the other hand, the cross section for exclusive diffractive production is known with sufficient accuracy to be sure that this channel can be used to play an important role in Higgs detection $^{11}$ via $H \rightarrow b \bar{b}$ at the LHC, provided that forward proton taggers are installed. The mass of the Higgs could then be accurately measured by the missing-mass method, $\Delta M_{\text {missing }} \simeq$ $1 \mathrm{GeV}[1]$. Moreover, the leading order $b \bar{b}$ background is strongly suppressed by a $J_{z}=0$ selection rule.

Details of the calculation of the $p p \rightarrow p+H+p$ exclusive Higgs production cross section are given in Ref. [16]. The cross section is predicted to be $3 \mathrm{fb}$ at the LHC, with a factor of two uncertainty [1]. The main sources of the $b \bar{b}$ background are, at leading order, caused by gluon jets being misidentified as a $b \bar{b}$ pair, by a $J_{z}=2$ admixture due to non-forward protons and by a $J_{z}=0$ contribution arising from $m_{b} \neq 0$. Also there is a background contribution from $b \bar{b} g$ events in which the emitted gluon is approximately collinear with a $b$ jet. These backgrounds were considered in detail in Ref. [1], leading to a signal-to-background ratio of about 3. Note that in [1] only the $g g \rightarrow b \bar{b} g$ hard subprocess was considered at NLO, and radiation for the spectator, screening gluon was not discussed. However, this latter process is numerically small because of the additional suppression of colour-octet $b \bar{b}$ production around $90^{\circ}$; rotational invariance around the $b$ quark direction causes the cross section to be proportional to $\cos ^{4} \theta$ in the $b \bar{b}$ c.m. frame [37].

We may summarize the exclusive diffractive Higgs signal $(p p \rightarrow p+H+p$ with $H \rightarrow b \bar{b})$ by the following example. Consider the detection of a Higgs of mass $120 \mathrm{GeV}$ with an integrated luminosity of $30 \mathrm{fb}^{-1}$ at the LHC. When account is taken of the efficiencies associated with proton tagging and with the identification of $b$ and $\bar{b}$ jets, and allowance is made for the polar angle cuts and the $H \rightarrow b \bar{b}$ branching ratio, then the original $(\sigma=3 \mathrm{fb}) \times\left(\mathcal{L}=30 \mathrm{fb}^{-1}\right)=90$ events is reduced to an observable signal of $11 H \rightarrow b \bar{b}$ events, with a background of 4 [1].

We stress that the predicted value of the exclusive cross section can be checked experimentally. All the ingredients, except for the NLO correction to the $g g \rightarrow H$ vertex, are the same for our signal as for exclusive double-diffractive dijet production, $p p \rightarrow p+\operatorname{dijet}+p$, where the dijet system is chosen in the same kinematic domain as the Higgs boson, that is $M(j j) \sim 120 \mathrm{GeV}$ $[16,11]$. Therefore by observing the larger dijet production rate, we can confirm, or correct, the estimate of the exclusive Higgs signal.

\footnotetext{
${ }^{11}$ Unfortunately, the cross section is too low for this method to be used at the Tevatron.
} 


\section{Acknowledgements}

We thank Brian Cox, Albert De Roeck, Rikard Enberg, Jeff Forshaw, Aliosha Kaidalov, Genya Levin, Leif Lönnblad, Risto Orava, Robi Peschanski, Christophe Royon, Torbjörn Sjöstrand and Michael Spira for useful discussions. One of us (VAK) thanks the Leverhulme Trust for a Fellowship. This work was partially supported by the UK Particle Physics and Astronomy Research Council, by the Russian Fund for Fundamental Research (grants 01-02-17095 and 00-15-96610) and by the EU Framework TMR programme, contract FMRX-CT98-0194 (DG 12-MIHT). 


\section{References}

[1] A. De Roeck, V.A. Khoze, A.D. Martin, R. Orava and M.G. Ryskin, hep-ph/0207042.

[2] M.G. Albrow and A. Rostovtsev, hep-ph/0009336.

[3] CDF Collaboration: T. Affolder et al., Phys. Rev. Lett. 84 (2000) 5043.

[4] V.A. Khoze, A.D. Martin and M.G. Ryskin, Phys. Lett. B502 (2001) 87.

[5] B.E. Cox, J.R. Forshaw and B. Heinemann, Phys. Lett. B540 (2002) 263.

[6] M. Boonekamp, R. Peschanski and C. Royon, Phys. Rev. Lett. 87 (2001) 251806.

[7] M. Boonekamp, A. De Roeck, R. Peschanski and C. Royon, hep-ph/0205332.

[8] R.B. Appleby and J.R. Forshaw, Phys. Lett. B541 (2002) 108.

[9] G. Ingelman and P.E. Schlein, Phys. Lett. B152 (1985) 256.

[10] M.A. Kimber, A.D. Martin and M.G. Ryskin, Eur. Phys. J. C12 (2000) 655;

Phys. Rev. D63 (2001) 114027.

[11] V.A. Khoze, A.D. Martin and M.G. Ryskin, Eur. Phys. J. C14 (2000) 525.

[12] V.A. Khoze, A.D. Martin and M.G. Ryskin, Eur. Phys. J. C18 (2000) 167.

[13] A.B. Kaidalov, V.A. Khoze, A.D. Martin and M.G. Ryskin, Eur. Phys. J. C21 (2001) 521.

[14] E. Gotsman, E. Levin and U. Maor, Phys. Rev. D60 (1999) 094011 and references therein.

[15] V.A. Khoze, A.D. Martin and M.G. Ryskin, Nucl. Phys. (Proc. Suppl.) B99 (2001) 213.

[16] V.A. Khoze, A.D. Martin and M.G. Ryskin, Eur. Phys. J. C23 (2002) 311.

[17] A. Bialas and P.V. Landshoff, Phys. Lett. B256 (1991) 540.

[18] F.E. Low, Phys. Rev. D12 (1975) 163;

S. Nussinov, Phys. Rev. Lett 34 (1976) 1286.

[19] R. Enberg, G. Ingelman, A. Kissavos and N. Timneanu, Phys. Rev. Lett. 89 (2002) 081801.

[20] E.M. Levin, hep-ph/9912402; and references therein.

[21] J.-R. Cudell and O.F. Hernandez, Nucl. Phys. B471 (1996) 471.

[22] A.D. Martin, M.G. Ryskin and V.A. Khoze, Phys. Rev. D56 (1997) 5867.

[23] A. Berera, Phys. Rev. D62 (2000) 014015. 
[24] A. Berera and J.C. Collins, Nucl. Phys. B474 (1996) 183.

[25] CDF Collaboration: T. Affolder et al., Phys. Rev. Lett., 85 (2000) 4215.

[26] V.A. Khoze, A.D. Martin and M.G. Ryskin, hep-ph/0006005, in Proc. of 8th Int. Workshop on Deep Inelastic Scattering and QCD (DIS2000), Liverpool, eds. J. Gracey and T. Greenshaw (World Scientific, 2001), p.592.

[27] V.A. Khoze, A.D. Martin and M.G. Ryskin, Phys. Lett. B401 (1997) 330.

[28] A.D. Martin and M.G. Ryskin, Phys. Rev. D64 (2001) 094017.

[29] A.G. Shuvaev, K.J. Golec-Biernat, A.D. Martin and M.G. Ryskin, Phys. Rev. D60 (1999) 014015 .

[30] H1 Collaboration: C. Adloff et al., Z. Phys. C76 (1997) 613.

[31] A. Donnachie and P.V. Landshoff, Phys. Lettt. B296 (1992) 227.

[32] D0 Collaboration: B. Abbott et al., Phys. Lett. B440 (1998) 189;

CDF Collaboration: F. Abe et al., Phys. Rev. Lett. 80 (1998) 1156; ibid., 81 (1998) 5278.

[33] R. Enberg et al., Phys. Rev. D64 (2001) 114015.

[34] T. Sjöstrand et al., Comput. Phys. Commun. 135 (2001) 238,

T. Sjöstrand, Comput. Phys. Commun. 82 (1994) 74.

[35] B.I. Ermolaev, V.S. Fadin and L.N. Lipatov, Sov. J. Nucl. Phys. 45 (1987) 508.

[36] V.A. Khoze, A.D. Martin and M.G. Ryskin, Eur. Phys. J. C19 (2001) 477, erratum C20 (2001) 599 .

[37] D.L. Borden, V.A. Khoze, W.J. Stirling and J. Ohnemus, Phys. Rev. D50 (1994) 4499. 


\begin{tabular}{|c|c|c|c|c|c|c|c|}
\hline \multirow{2}{*}{ Reference } & \multirow{2}{*}{ Process } & \multicolumn{2}{|c|}{ Survival factor } & \multirow{2}{*}{ Norm. } & \multicolumn{2}{|c|}{$\sigma_{\text {Higgs }}(\mathrm{fb})$} & \multirow{2}{*}{ Notes } \\
\hline & & $T^{2}$ & $S^{2}$ & & Teva. & LHC & \\
\hline $\begin{array}{l}\text { Cudell, } \\
\text { Hernandez [21] }\end{array}$ & $\begin{array}{l}\text { excl } \\
\text { incl }\end{array}$ & no & no & $\sigma_{\text {tot }}$ & $\begin{array}{c}30 \\
200 \\
\end{array}$ & $\begin{array}{c}300 \\
1200 \\
\end{array}$ & $\begin{array}{l}\text { Overshoots CDF dijets } \\
\text { by } 1000 .\end{array}$ \\
\hline Levin [20] & $\begin{array}{l}\text { excl } \\
\text { incl }\end{array}$ & $\begin{array}{c}\text { yes } \\
\text { No DL }\end{array}$ & yes & $\sigma_{\text {tot }}$ & $\begin{array}{l}20 \\
70\end{array}$ & - & $\begin{array}{l}\text { Overshoots CDF dijets } \\
\text { by } 300 .\end{array}$ \\
\hline $\begin{array}{l}\text { Khoze, Martin, } \\
\text { Ryskin }[16]\end{array}$ & $\begin{array}{c}\text { excl } \\
\text { incl } \\
\text { C.inel } \\
\end{array}$ & yes & yes & $\begin{array}{l}\mathrm{pdf} \\
\mathrm{pdf}\end{array}$ & $\begin{array}{c}0.2 \\
1 \\
\sim 0.03\end{array}$ & $\begin{array}{c}3 \\
40 \\
50 \\
\end{array}$ & $\begin{array}{l}\text { Uses skewed gluons. } \\
\text { CDF dijets OK. }\end{array}$ \\
\hline $\begin{array}{l}\text { Cox, Forshaw, } \\
\text { Heinemann [5] }\end{array}$ & C.inel & $T \simeq 1$ & norm & $\begin{array}{l}\mathrm{CDF} \\
\text { dijet }\end{array}$ & 0.02 & 6 & $\begin{array}{l}\text { No LO, only NLO, QCD } \\
\text { i.e., no Fig. } 2(\mathrm{a}) \text {, only } 2(\mathrm{c})\end{array}$ \\
\hline $\begin{array}{l}\text { Boonekamp, } \\
\text { De Roeck, } \\
\text { Peschanski, } \\
\text { Royon [7] }\end{array}$ & C.inel & $T \simeq 1$ & norm & $\begin{array}{l}\mathrm{CDF} \\
\text { dijet }\end{array}$ & 2.7 & 320 & $\begin{array}{l}\text { No LO, only NLO, QCD. } \\
\text { Assume } S_{\mathrm{CDF}}^{2}=S_{\mathrm{LHC}}^{2}\end{array}$ \\
\hline $\begin{array}{l}\text { Enberg, } \\
\text { Ingelman, } \\
\text { Kissavos, } \\
\text { Timneanu [19] }\end{array}$ & $\begin{array}{l}\text { incl } \\
\text { C.inel }\end{array}$ & yes & yes & $F_{2}^{\text {Diff. }}$ & $<0.01$ & 0.2 & No coherence. \\
\hline
\end{tabular}

Table 1: Recent QCD-based calculations of the cross section, $\sigma_{\text {Higgs }}$, for exclusive, inclusive and Central inelastic double-diffractive production of a Higgs boson of mass about $120 \mathrm{GeV}$, at Tevatron and LHC energies. The Norm. column indicates the way in which the various predicted cross sections are normalised. $T^{2}$ and $S^{2}$ are the survival probabilities of the rapidity gaps to QCD radiation associated with the hard $g g \rightarrow H$ subprocess and to soft rescattering, respectively; "norm" in the $S^{2}$ column means that $S^{2}$ is simply determined by normalising to CDF dijet data [25]. The cross sections for central inelastic production (C.inel) correspond to integrating up to $M_{\text {miss }}=0.1 \sqrt{s}$, where $\sqrt{s}$ is the collider energy. Note that in Ref. [16] the C.inel cross section is $0.2 \mathrm{fb}$ at the Tevatron, but this includes the exclusive contribution. The LHC entry for Cox et al. [5] is obtained using $S^{2}=0.02$. 